Advances and Applications, Vol. 41

(C) 1989 Birkhäuser Verlag Basel

\title{
FACTORIZATION OF NONLINEAR SYSTEMS *
}

\author{
J. William Helton
}

Dedicated to Israel Gohberg on his sixtieth birthday

A basic result of Bart, Gohberg, Kaashoek, and Van Dooren parameterizes factorizations of the transfer function of a linear system in terms of the state space equations of the system. This paper extends their result to nonlinear systems.

\section{§1. INTRODUCTION}

When one system $S_{1}$ has output feeding into another system $S_{2}$ this produces another system called the product of two systems. The reverse procedure of factoring a given system into simpler systems is a classic tool of linear circuits, filters, and now control; indeed, it is one of the most powerful tools in theoretical engineering.

Even in the nonlinear case it has been recently shown that when factorizations of various types are possible stability results $[\mathrm{Hm}],[\mathrm{AD}]$, and $H^{\infty}$ control type results $[\mathrm{BH}]$ are possible. These factorizations often are not minimal in the sense that the dimension of the state spaces of the factors of the system sum to the dimension of the state space of the system.

This paper analyzes the existence, construction, uniqueness and properties of minimal factorizations for nonlinear systems. While factorization in terms of frequency response functions goes back at least to Cauer in the 1930's, the classic state space result is due to Bart, Gohberg, Kaashoek and Van Dooren (cf. [BGK]). For a linear $A B C D$ system with $D$ invertible it sets minimal factorizations into direct correspondence with

* Research on this paper was supported in part by the National Science Foundation and the Air Force Office of Scientific Research. 
a pair $\mathcal{S}, \mathcal{S}^{\times}$of disjoint subspaces of the state space $X$ which actually decompose $X$ as $X=\mathcal{S}+\mathcal{S}^{\times}$and which have the distinctive properties that

(1.1) $\mathcal{S}$ is invariant under $A$.

$\left(1.1^{\times}\right) \mathcal{S}^{\times}$is invariant under $A^{\times} \triangleq A-B D^{-1} C$.

In Sections 2 and 3 we generalize this to nonlinear systems. The nonlinear state space $X$ will be a manifold. While $\mathcal{S}, \mathcal{S}^{\times}$generalize to a particular set of coordinates $\left(X_{1}, X_{2}\right)$ on the manifold $x^{1} \in \mathcal{S}, x^{2} \in \mathcal{S}^{\times}$. These must be invariant in a natural sense which we describe explicitly.

In Section 4 we show that generically invariant coordinates are uniquely determined (and very likely overdetermined) by very little information--namely the linearization at an equilibrium point. Clearly, if systems $S_{1}, S_{2}$ have the same equilibrium points $e_{1}, e_{2}$ their product has an equilibrium point $e$. Also the product $L$ of linearizations $L_{1}$, $L_{2}$ about $e_{1}, e_{2}$ equals the linearization of the product $S_{1}, S_{2}$ about $e_{1}, e_{2}$. Our results on $\mathcal{S}, \mathcal{S}^{\times}$decompositions lead us to conjecture that

generically at most one 'nontrivial' smooth minimal nonlinear factorization corresponds to each factorization of the linearized system, $L$.

Incleed for a particular factorization of $L$ to come from nonlinear factors of $S$ requires that a compatibility condition be satisfied. This article actually proves this except for one gap (Conjecture 4.5). Various experts in nonlinear ordinary differential equations assured us that Conjecture 4.5 is true; but they do not know a precise reference for it in the literature. In this paper factorization typically means minimal factorization.

The author wishes to thank Chris Byrnes for helpful discussions and Asher BenArtzi for comments on the manuscript. The proof presented here of Lemma 2.4 was given by Ben Artzi. Neola Crimmins cheerfully typed the manuscript from a rugged original. 


\section{§2. FACTORIZATION AND $\hat{F}, \hat{G}$ INVARIANT COORDINATES; DISCRETE TIME}

This section extends the classic result of Bart, Gohberg, Kaashoek, and Van Dooren: on state space description of factorization mentioned in the Introduction. First we treat discrete time systems; second continuous time systems.

We start with a system $\hat{F}, \hat{G}$ which is the product of systems $f, g$ and $F, G$

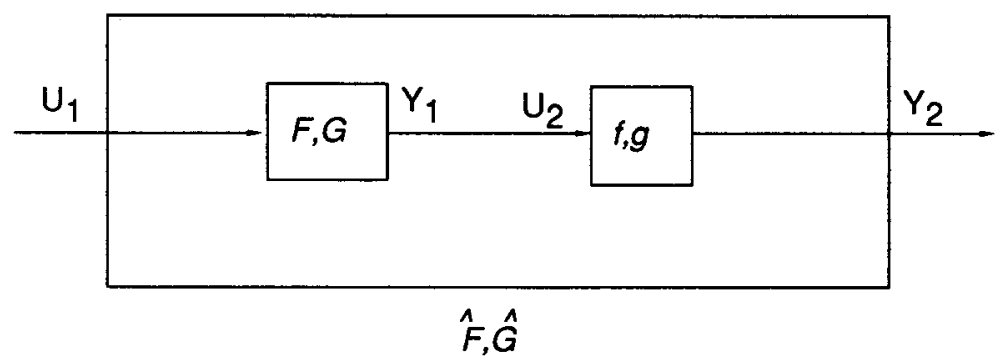

Figure 2.1.

and give a list of properties which $\hat{F}, \hat{G}$ must have as a result of the factorization. Later we go backwards.

Our notation is:

$X_{1}, X_{2}$ are finite dimensional manifolds.

$U_{1}, U_{2}$ are finite dimensional vector spaces.

$Y_{1}, Y_{2}$ are finite dimensional vector spaces.

$$
\begin{aligned}
& F: X_{1} \times U_{1} \longrightarrow X_{1}, \quad G: X_{1} \times U_{1} \longrightarrow Y_{1} . \\
& f: X_{2} \times U_{2} \longrightarrow X_{2} \quad g: X_{2} \times U_{2} \longrightarrow Y_{2} .
\end{aligned}
$$


We always assume special properties of our systems. First we assume $Y_{1}=U_{2}$. We also assume throughout that $\operatorname{dim} U_{1}=\operatorname{dim} Y_{1}=\operatorname{dim} U_{2}=\operatorname{dim} Y_{2}$, and that for each $x^{2} \in X_{1}$ the map $G\left(x^{1}, \cdot\right): U_{1} \rightarrow Y_{1}$ is invertible with inverse denoted by $G^{I}\left(x^{1}, \cdot\right)$. Of course

$$
G\left(x^{1}, G^{I}\left(x^{1}, y\right)\right)=y \quad G^{I}\left(x^{1}, G\left(x^{1}, u\right)\right)=y
$$

Likewise for each $x^{2} \in X_{2}$, we have $g\left(x^{2}, \cdot\right)$ invertible with inverse denoted $g^{I}\left(x^{2}, \cdot\right)$. Call such a system $F, G$ an invertible system. For a system $F, G$ denote by $F^{\times}: X \times Y_{1} \rightarrow X_{1}$ the map

$$
F^{\times}\left(x^{1}, y\right)=F\left(\left(x^{1}, G^{I}\left(x^{1}, y\right)\right)\right.
$$

for $x^{1} \in X_{1}, y \in Y_{1}$. The key property of $F^{\times}$is

LEMMA 2.1. The system $F^{\times}, G^{I}$ is the inverse of $F, G$.

PROOF. By definition of $G^{I}$

$$
y=G(x, u) \quad u=G^{I}(x, y) .
$$

Therefore

$$
x_{n+1}=F\left(x_{n}, u_{n}\right) \quad y_{n}=G\left(x_{n}, u_{n}\right)
$$

is equivalent to

$$
\begin{aligned}
x_{n+1} & =F\left(x_{n}, G^{I}\left(x_{n}, y_{n}\right)\right)=F^{\times}\left(x_{n}, y_{n}\right) \\
u_{n} & =G^{I}\left(x_{n}, y_{n}\right) .
\end{aligned}
$$

We say that $e^{1}$ (resp. $e^{2}$ ) is an equilibrium point for $F, G$ (resp. $f, g$ ) provided that

$$
F\left(e^{1}, 0\right)=e^{1}
$$

respectively,

$$
f\left(e^{2}, G\left(e^{\prime}, 0\right)\right)=e^{2} .
$$

Naturally our system dynamics are:

$$
\begin{aligned}
x_{n+1}^{1}=F\left(x_{n}^{1}, u_{n}^{1}\right) \quad & x_{n+1}^{n}=f\left(x_{n}^{2}, u_{n}^{2}\right) \\
& y_{n}^{1}=G\left(x_{n}^{1}, u_{n}^{1}\right) \quad y_{n}^{2}=g\left(x_{n}^{2}, u_{n}^{2}\right)
\end{aligned}
$$


and if one system is plugged into the other, that is $y_{n}^{1}=u_{n}^{2}$, we have that the product system acts on state manifold

$$
X=\left\{\left(x^{1}, x^{2}\right): x^{j} \in X_{j}\right\}
$$

and is $\hat{F}, \hat{G}$ given by

$$
\begin{gathered}
\hat{F}\left(\left(x^{1}, x^{2}\right), u\right)=\left(F\left(x^{1}, u\right), f\left(x^{2}, G\left(x^{1}, u\right)\right)\right) \\
\hat{G}\left(\left(x^{1}, x^{2}\right), u\right)=g\left(x^{2}, G\left(x^{1}, u\right)\right)
\end{gathered}
$$

for $\left(x^{1}, x^{2}\right) \in X$ and input $u \in U_{1}$. Note that invertibility of systems (2.1) implies invertibility of the system $\hat{F}, \hat{G}$.

We now write down properties of the system $\hat{F}, \hat{G}$ defined by (2.3) which arise because it is a product system, but which do not mention $F, G, f, g$ explicitly. Indeed

$$
\hat{F}: X \times U_{1} \longrightarrow X \quad \hat{G}: X \times U_{2} \longrightarrow Y_{2}
$$

The state space $X$ has a coordinate pair ${ }^{1} X_{1}, X_{2}$ so that $X=\left\{\left(x^{1}, x^{2}\right): x^{j} \in X_{j}\right\}$ with the properties for $\left(x^{1}, x^{2}\right) \in X, u \in U_{1}$

(2.4) $\pi^{1} \hat{F}\left(\left(x^{1}, x^{2}\right), u\right)$ is independent of $x^{2}$.

$\left(2.4^{\times}\right)^{2} \quad \pi^{2} \hat{F}^{\times}\left(\left(x^{1}, x^{2}\right), y\right)$ is independent of $x^{1}$.

(2.5a) $\hat{G}^{I}((a, c), \hat{G}((b, c), u))$ is independent of $c$.

(2.5b) $\hat{G}\left((a, c), \hat{G}^{I}((a, d), y)\right)$ is independent of $a$.

Here $\pi^{j}$ denotes the projection $\pi^{j}\left(x^{1}, x^{2}\right)=x^{j}$.

If $e_{1}, e_{2}$ are equilibrium points for the factors $F, G$ and $f, g$, then

(2.6) $e=\left(e^{1}, e^{2}\right)$ is an equilibrium point for $\hat{F}, \hat{G}$ and consequently

$$
\hat{F}^{\times}(e, \hat{G}(e, 0))=\hat{F}(e, 0)=e .
$$

1 By coordinate pair we mean direct product; there is a $C^{\infty}$ diffeomorphism $\varphi: X_{1} \times$ $X_{2} \rightarrow X$. The (important) case where $\varphi$ is not $1-1$ is postponed.

${ }^{2}$ Actually the stronger statement: For any $a, b \in X_{2}$ the quantity $\pi^{2} \hat{F}\left(\left(x^{1}, a\right)\right.$, $\left.G^{I}\left(\left(x^{1}, b\right), y\right)\right)$ is independent of $x^{1}$ holds. When $a=b$ this gives $\left(2.4^{\times}\right)$. 
Here $\pi^{j}$ denotes the projection $\pi^{j}\left(x^{1}, x^{2}\right)=x^{j}$.

Now we consolidate this abstraction. Suppose we are: Given a system $\hat{F}, \hat{G}$, with state manifold $X$, and input, output spaces $U, Y$. We say that $\left(X_{1}, X_{2}\right)$ is a product coordinate pair for the system $\hat{F}, \hat{G}$ provided that (2.4) and (2.5) are satisfied.

THEOREM 2.1. The invertible system $\hat{F}, \hat{G}$ has factors $F, G$ and $f, g$ which are invertible systems if $X$ has a product pair. Each product pair $\left(X_{1}, X_{2}\right)$ determines the dynamics $F$ and $f^{\times}$of the factors uniquely.

Conversely, suppose $\hat{F}, \hat{G}$ has state manifold $X$ of dimension $\hat{N}$. Formulas for factors are given in Recipe 2.3. The factors have an equilibrium point $e^{1}, e^{2}$ if $\hat{F}, \hat{G}$ has an equilibrium point $e$ in the sense of (2.6). The converse is true in a local sense to be discussed in Theorem 2.4 .

The key product pair conditions $(2.4)$ and $\left(2.4^{\times}\right)$are easy to visualize. Fix $b \in X_{2}, a \in X_{1}$; we think of $\left(X_{1}, b\right)$ and $\left(a, X_{2}\right)$ as leaves of the $x$-coordinate foliations. Condition (2.4) says precisely that for any $u \in U_{1}$

$$
\hat{F}\left(\left(a, X_{2}\right), u\right)=\left(\tilde{a}, \tilde{X}_{2}\right) \subset\left(\tilde{a}, X_{2}\right)
$$

where for each fixed $a$, one gets a fixed $\tilde{a} \in X_{1}$ and $\tilde{X}_{2}$ a subset of $X_{2}$. Likewise $\left(2.4^{\times}\right)$is equivalent to

$$
\hat{F}^{\times}\left(\left(X_{1}, b\right), y\right) \subset\left(X_{1}, \tilde{b}\right)
$$

for any $b, y$ and some $\tilde{b}$. Note that when $\hat{F}, \hat{G}$ is a linear system and $X_{1}, X_{2}$ are linear spanning nonintersecting subspaces, then condition (2.4a) says

$$
\begin{aligned}
\hat{F}\left(\left(a, X_{2}\right), u\right) & =\hat{A}(a, 0)+\hat{A}\left(0, X_{2}\right)+\hat{B} u \\
& \subset(\tilde{a}, 0)+\left(0, X_{2}\right)
\end{aligned}
$$

So $\hat{A}(a, 0)+\hat{B} u=\left(\hat{a}, \tilde{x}_{2}\right)$ and $\hat{A}\left(0, X_{2}\right) \subset\left(0, X_{2}\right)$. That is, $\left(0, X_{2}\right)$ is an invariant subspace for $\hat{A}$. Likewise $\left(X_{1}, 0\right)$ is invariant for $A^{\times}$so conditions (2.4) generalize those of [BGK] and $[\mathbf{v D}]$.

LEMMA 2.2. $\hat{G}^{I}\left(\left(x^{1}, x^{2}\right), y\right)=G^{I}\left(x^{1}, g^{I}\left(x^{2}, y\right)\right)$. 


$$
\begin{aligned}
& \text { PROOF. } y=\hat{G}\left(\left(x^{1}, x^{2}\right), u\right) \triangleq g\left(x^{2}, G\left(x^{1}, u\right)\right) \text {. } \\
& g^{I}\left(x^{2}, \hat{G}\left(\left(x^{1}, x^{2}\right), u\right)=G\left(x^{1}, u\right)\right. \\
& G^{I}\left(x^{1}, g^{I}\left(x^{2}, \hat{G}\left(\left(x^{1}, x^{2}\right), u\right)\right)=u\right. \text {. }
\end{aligned}
$$

Since $y=\hat{G}\left(\left(x^{1}, x^{2}\right), u\right)$ we have $G^{I}\left(x^{1}, g^{I}\left(x^{2}, y\right)\right)=u$ which proves the lemma.

Theorem 2.1 is proved by the following construction:

RECIPE 2.3. Given $\hat{F}, \hat{G}$ and a product coordinate pair $\left(X_{1}, X_{2}\right)$ for it. First ${ }^{3}$ select $c_{1} \in X^{1}$ and $\varphi$ a diffeomorphism from $U^{2}$ onto $U^{2}$. All factors of $\hat{F}, \hat{G}$ are given by

$$
g\left(x^{2}, u\right)=\hat{G}\left(\left(c^{1}, x^{2}\right), \varphi^{-1}(u)\right)
$$

$$
G\left(x^{1}, u\right)=\varphi \circ \hat{G}^{I}\left(\left(c^{1}, c^{2}\right), \hat{G}\left(\left(x^{1}, c^{2}\right), u\right)\right) \text { independent of } c^{2}
$$

$$
F\left(x^{1}, u\right)=\pi^{1} \hat{F}\left(\left(x^{1}, \beta\right), u\right) \text { independent of } \beta
$$

$$
\begin{aligned}
f\left(\left(x^{2}, u^{2}\right)\right) & =\pi^{2} \hat{F}\left(\left(\xi, x^{2}\right), \hat{G}^{I}\left(\left(\xi, x^{2}\right), g\left(x^{2}, u^{2}\right)\right) \text { independent of } \xi\right. \\
& \triangleq \pi^{2} \hat{F}^{\times}\left(\left(\xi, x^{2}\right), g\left(x^{2}, u^{2}\right)\right)
\end{aligned}
$$

${ }^{3}$ There appears to be an asymmetry between $c^{1}$ and $c^{2}$ in (2.7) and (2.8). An alternative formula for $g$ and $G$ dispels this illusion.

First select $c^{1} \in X^{1} c^{2} \in X^{2}$. Next select two maps

$$
\delta^{2}: U^{2}=Y^{1} \rightarrow Y^{2} \quad \delta^{1}: U^{2}=Y^{1} \rightarrow U^{1}
$$

which factor $\hat{G}\left(\left(c^{1}, c^{2}\right),\right)$ in the sense

$$
\delta^{2} \circ \delta^{1^{-1}}(u)=\hat{G}\left(\left(c^{1}, c^{2}\right), u\right) \text { for all } u \in U_{1}
$$

Define

$\left(2.7^{\prime}\right) \quad g^{I}\left(x^{2}, y\right)=\delta^{2^{-1}} \circ \hat{G}\left(\left(\tau, c^{2}\right), \hat{G}^{I}\left(\left(\tau, x^{2}\right), y\right)\right)$ independent of $\tau$.

$\left(2.8^{\prime}\right) \quad G\left(\left(x^{1}, u\right)\right)=\delta^{1^{-1}} \circ \hat{G}^{I}\left(\left(c^{1}, \rho\right), \hat{G}\left(x^{1}, \rho, u\right)\right)$ independent of $\rho$. 
The indepenclences follow from $(2.5 \mathrm{a}),(2.4)$ and $\left(2.4^{\times}\right)$, respectively.

REMARK 2.3. A particular coordinate pair $\left(X_{1}, X_{2}\right)$ determines $F$ uniquely from $\hat{F}$, via equation (2.9). Liliewise $\left(X_{1}, X_{2}\right)$ determines $f^{\times}\left(x^{2}, y^{2}\right)=\pi^{2} \hat{F}^{\times}\left(\left(\xi, x^{2}\right), y^{2}\right)$ uniquely.

PROOF OF THEOREM 2.1. We must prove that composition of these systems yields $\hat{F}, \hat{G}$, that is

$$
\begin{gathered}
\pi_{1} \hat{F}\left(\left(x^{1}, x^{2}\right), u\right)=F\left(x^{1}, u\right), \\
\pi_{2} \hat{F}\left(\left(x^{1}, x^{2}\right)=f\left(x^{2}, G\left(x^{1}, u\right)\right),\right. \\
\hat{G}\left(\left(x^{1}, x^{2}\right), u\right)=g\left(x^{2}, G\left(x^{1}, u\right)\right),
\end{gathered}
$$

and that these are the only possibilities.

Equation (2.13) and non-uniqueness of factors is handled by

\section{LEMMA 2.4. The equation}

$$
\hat{G}\left(\left(x^{1}, x^{2}\right), u\right)=g\left(x^{2}, G\left(x^{1}, u\right)\right)
$$

admits a solution $(g, G)$ if and only if $(2.5 \mathrm{a})$ holds, then one solution is given by

$$
g=g_{1}\left(x^{2}, u\right)=\hat{G}\left(\left(c^{1}, x^{2}\right), u\right) G=G_{1}\left(x^{1}, u\right)=\hat{G}^{I}\left(\left(c^{1}, c^{2}\right), \hat{G}\left(\left(x^{1}, c^{2}\right), u\right),\right.
$$

where $c^{1} \in X^{1}$ and $c^{2} \in X^{2}$ are arbitrary. The most general solution of equality (2.14) is given by

$$
g\left(x^{2}, u\right)=g_{1}\left(x^{2}, \varphi^{-1}(u)\right) G\left(x^{1}, u\right)=\varphi\left(G_{1}\left(x^{1}, u\right)\right)
$$

where $\varphi: U_{2} \rightarrow U_{2}$ is an arbitrary diffeomorphism.

PROOF. Assume that (2.14) holds, and denote $z=\hat{G}^{I}((a, c), \hat{G}((b, c), u))$. Then $\hat{G}((a, c), z)=\hat{G}((b, c), u)$. By (2.14), this leads to $g(c, G(a, z))=g(c, G(b, u))$. 
Applying $g^{1}(c, \cdot)$ on both sides of this equality, we obtain $G(a, z)=G(b, a)$. Thus $z=G^{I}(a, G(b, u))$ and therefore $z$ is independent of $c$. Hence (2.5a) holds.

Assume now that (2.5a) holds. Then

$$
\hat{G}^{I}\left(\left(c^{1}, c^{2}\right), \hat{G}\left(\left(x^{1}, c^{2}\right), u\right)=\hat{G}^{I}\left(\left(c^{1}, x^{2}\right), \hat{G}\left(\left(x^{1}, x^{2}\right), u\right)\right.\right.
$$

for every $\left(x^{1}, x^{2}, u\right) \in x_{1} \times x_{2} \times u_{1}$. We now apply $\hat{G}\left(\left(c^{1}, x^{2}\right), \cdot\right)$ on both sides of this equality and obtain

$$
\hat{G}\left(\left(c^{1}, x^{2}\right), \hat{G}^{I}\left(\left(c^{1}, c^{2}\right), \hat{G}\left(\left(x^{1}, c^{2}\right), u\right)\right)\right)=\hat{G}\left(\left(c^{1}, x^{2}\right), \hat{G}^{I}\left(\left(c^{1}, x^{2}\right), \hat{G}\left(\left(x^{1}, x^{2}\right), u\right)\right)\right)
$$

However the left hand side of this equality is equal to $g_{1}\left(x^{2}, G_{1}\left(x^{1}, u\right)\right)$, while the right hand side is equal to $\hat{G}\left(\left(x^{1}, x^{2}\right), u\right)$. Therefore (2.15) gives a solution of (1).

Let $\varphi: U_{2} \rightarrow U_{2}$ be a diffeomorphism and let $g, G$ be given by (3). Clearly, using the fact that (2) gives a solution, we have

$$
g\left(x^{2}, G\left(x^{1}, u\right)\right)=g_{1}\left(x^{2}, \varphi^{-1}\left(\varphi\left(G_{1}\left(x^{1}, u\right)\right)\right)\right)=g_{1}\left(x^{2}, G_{1}\left(x^{1}, u\right)\right)=\hat{G}
$$

Finally, assume that $(g, G)$ and $\left(g_{1}, G_{1}\right)$ are arbitrary solutions of (1).

$$
g\left(x^{2}, G\left(x^{1}, u\right)\right)=g_{1}\left(x^{2}, G_{1}\left(x^{1}, u\right)\right) .
$$

Therefore $G\left(x^{1}, u\right)=g^{I}\left(x^{2}, g_{1}\left(x^{2}, G_{1}\left(x^{1}, u\right)\right)\right)$. Fixing $x^{2}=c^{2}$, and denoting $\varphi(\cdot)=$ $g^{I}\left(c^{2}, g_{1}\left(c^{2}, \cdot\right)\right)$, we obtain

$$
G\left(x^{1}, u\right)=\varphi\left(G_{1}\left(x^{1}, u\right)\right)
$$

where $\varphi: U_{2} \rightarrow U_{2}$ is a diffeomorphism. Inserting the last equality in (2.17) we obtain

$$
g\left(x^{2}, \varphi\left(G_{1}\left(x^{1}, u\right)\right)=g_{1}\left(x^{2}, G_{1}\left(x^{1}, u\right)\right)\right.
$$

Let $y \in U_{2}$, there is a $u$ such that $y=\varphi\left(G_{1}\left(x^{1}, u\right)\right)$, and therefore

$$
g\left(x^{2}, y\right)=g_{1}\left(x^{2}, G_{1}\left(x^{1}, u\right)\right)=g_{1}\left(x^{2}, \varphi^{-1}(y)\right)
$$

The equalities (2.16) follow from (2.18) and (2.19). 
We prove (2.12):

$$
\begin{aligned}
f\left(x^{2}, G\left(x^{1}, u\right)\right) & =\pi^{2} \hat{F}\left(\left(\xi, x^{2}\right), \hat{G}^{I}\left(\left(\xi, x^{2}\right), g\left(x^{2}, G\left(x^{1}, u\right)\right)\right.\right. \\
& =\pi^{2} \hat{F}\left(\left(\xi, x^{2}\right), \hat{G}^{I}\left(\left(\xi x^{2}\right), \hat{G}\left(\left(x^{1}, x^{2}\right), u\right)\right)\right.
\end{aligned}
$$

first by definition (2.10) and then by (2.13). Independence of $\xi$ implies we may take $\xi=x^{1}$ to get

$$
f\left(x^{2}, G\left(x^{1}, u\right)\right)=\pi^{2} \hat{F}\left(\left(x^{1}, x^{2}\right), u\right)
$$

as desired.

Suppose that $e$ is an equilibrium point of $\hat{F}$, that is, $\hat{F}(e, 0)=e$; let $e^{j}=\pi^{j} e$. Then by (2.9)

$$
F\left(e^{1}, 0\right)=\pi^{1} \hat{F}\left(\left(e^{1}, \beta\right), 0\right)=\pi^{1} \hat{F}(e, 0)=0
$$

since we may take $\beta=e^{2}$. By (2.10) and then (2.6),

$$
\begin{aligned}
f\left(e^{2}, G\left(e^{1}, 0\right)\right) & =\pi^{2} \hat{F}\left(e, \hat{G}^{I}\left(\left(\xi, e^{2}\right), g\left(e^{2}, G\left(e^{1}, 0\right)\right)\right)\right. \\
& =\pi^{2} \hat{F}\left(\xi, e^{2}\right), \hat{G}^{I}\left(e, g\left(e^{2}, G\left(e^{1}, 0\right)\right)\right) \\
& =\pi^{2} \hat{F}\left(e, \hat{G}^{I}(e, \hat{G}(e, 0))\right)=\pi^{2} \hat{F}^{x}(e, \hat{G}(e, 0)) \\
& \pi^{2} e=e^{2} .
\end{aligned}
$$

Now we change directions and treat the converse of Theorem 2.1. There are generally speaking two natural notions of factoring a system $\hat{F}, \hat{G}$ and since we are operating at a greater level of detail, we will give three.

(F1) $\hat{F}, \hat{G}$ is the product of $F, G$ and $f, g$ as in (2.3).

(F2) The transfer operators ${ }^{4}$ for the systems satisfy $\hat{\Phi}=\phi \circ \Phi$.

(F3) The product of systems $F, G$ and $f, g$ as in (2.3) which we now denote by $\tilde{F}, \tilde{G}$ is not minimal and $\hat{F}, \hat{G}$ is a restriction of it, more precisely there is a map $\psi: X_{1} \times X_{2} \rightarrow X$ which is smooth, onto and whose differential has constant rank, such that

${ }^{4}$ The transfer operator $\phi$ of a system $(f, g)$ with equilibrium point $e$ is a mapping from the space $\ell^{+}$of sequences $\left\{\left(u_{0}, u_{1} \ldots\right\}\right.$ to $\ell^{+}$defined by $\phi\left(\left(u_{0}, u_{1}, \ldots\right)\right)=\left(y_{0}, y_{1}, \ldots\right)$ where the $y$ 's are produced by recursion (2.1) defining system $f, g$ when $x_{0}=e$ and the $u_{j}$ are fed to the system. The definitions of $\hat{\Phi}$ and $\Phi$ are the same. 


$$
\begin{array}{ccc}
\hat{F}(\psi(x), u)=\psi \circ \tilde{F}(x, u) & \forall x \in X_{1} \times X_{2} \\
\hat{G}(\psi(x), u) & =\tilde{G}(x, u) & \forall u \in U .
\end{array}
$$

These definitions are related. In particular if systems $\hat{F}, \hat{G}$ and $f, g$ are well behaved, controllable, and reachable in a strong sense one expects that (F2) and (F3) are equivalent at least locally. Since this type of equivalence is not the subject of this paper we henceforth treat only factorization of type (F1) and of type (F3). The reader may intuitively bear in mind that all three types are closely related.

Suppose that $\hat{F}, \hat{G}$ and $X$ factors in sense (F3). We know that there is a system $\tilde{F}, \tilde{G}$ on state manifold $\tilde{X}_{1} \times \tilde{X}_{2}$ and a smooth map $\psi: \tilde{X}_{1} \times \tilde{X}_{2} \rightarrow X$ satisfying (2.20). Also we know that $\tilde{F}, \tilde{G}$ is built from factors as in (F1) and so $\tilde{X}_{1}, \tilde{X}_{2}$ is a coordinate pair for $\tilde{F}, \tilde{G}$. Suppose that $X$ and $\tilde{X}_{1} \times \tilde{X}_{2}$ have the same dimension and $\psi$ is nondegenerate, onto. Then the map $\psi$ is a diffeomorphism locally on $\tilde{X}_{1} \times \tilde{X}_{2}$, while it may not be one globally (e.g. if it is a covering map). Thus the fact that $\hat{F}, \hat{G}$ factors shows up in an awkward way on its state manifold, and we leave sorting it out as an open question.

However, locally many situations are well behaved. Intuitively if the discrete system comes from a continuous time system and the discretizing time step is small enough, then locally $X$ must have product pairs for which (2.3) holds at least for small $u$.

THEOREM 2.4. Suppose that $\hat{F}, \hat{G}$ factors in sense (F3) and that the dimensions of the state manifolds of the factors sum to $\hat{N}$ the dimension of the state manifold of $\hat{F}, \hat{G}$. Given $x \in X$, there is a neighborhood $\mathcal{O}$ of $x$ which possesses a product pair $\tilde{X}_{1} \times \tilde{X}_{2}$. This is in the sense that $\mathcal{O} \subset \tilde{X}_{1} \times \tilde{X}_{2}$, and if $\mathcal{O}_{0}$ is open inside $\mathcal{O}$ and $0 \in U_{0}$ (resp. $y \in Y_{0}$ ) is a neighborhood in $U$ (resp. $Y$ ) with the property that $\hat{F}, \pi_{1} \hat{F}, \pi_{2} \hat{F}^{\times}$map $\mathcal{O}_{0} \times U_{0}$ into $\mathcal{O}$, then $(2 ., 4),\left(2.4^{\times}\right)$and $(2.5)$ hold for all $\left(x_{1}, x_{2}\right),(a, c),(b, c)(a, d) \in \mathcal{O}_{0}$ and $u \in U_{0}$ and $y \in Y_{0}$.

PROOF OF THEOREM 2.4. First we treat the global case. Suppose that $\hat{F}, \hat{G}$ factors in the sense (F1). We prove that factors $F, G$ and $f, g$ produce an $\tilde{F}, \tilde{G}$ with properties (2.4), (2.5) and (2.6). Each equation takes only a few lines. 
(2.4) is obvious.

$$
\begin{aligned}
\pi^{2} \hat{F}^{\times}\left(\left(x^{1}, a\right), y\right) & =\pi_{2} \hat{F}\left(\left(x^{1}, a\right), \hat{G}^{I}\left(\left(x^{1}, b\right), y\right)\right) \\
& =f\left(a,\left(G\left(x^{1}, G^{I}\left(x^{1}, g^{I}(b, y)\right)\right)=f\left(a, g^{I}(b, y)\right)\right.\right.
\end{aligned}
$$

which is independent of $x^{1}$.

$$
\left.\hat{G}^{I}((a, c), \hat{G}((b, c), u))\right)=G^{I}\left(a, g^{I}\left(c, g(c, G(b, u))=G^{I}(a, G(b, u))\right.\right.
$$

which is independent of $c$.

$$
\hat{G}\left((a, c), \hat{G}^{I}((a, d), y)\right)=g\left(c, G\left(a, G^{I}\left(\left(a, g^{I}(d, y)\right)=g\left(c, g^{I}(d, y)\right)\right.\right.\right.
$$

which is independent of $a$.

Note the equilibrium point condition (2.6) is true because

$$
\hat{F}^{\times}(e, \hat{G}(e, 0))=\hat{F}\left(e, \hat{G}^{I}(e, \hat{G}(e, 0))=\hat{F}(e, 0)=e .\right.
$$

The fact that $\hat{N}=N+n$ forces $\varphi$ to be a local diffeomorphism of the global case we just treated onto the state manifold of the given system. It is straightforward to obtain the theorem from this.

\section{§3. CONTINUOUS TIME}

Our notation is

$$
\begin{array}{ll}
\frac{d x^{1}}{d t}=F\left(x^{1}, u^{1}\right) & y^{1}=G\left(x^{1}, u^{1}\right) \\
\frac{d x^{2}}{d t}=f\left(x^{2}, u^{2}\right) & y^{2}=g\left(x^{2}, u^{2}\right)
\end{array}
$$

with $X_{1}, X_{2}$ manifolds $U_{1}, U_{2}=Y_{1}, Y_{2}$ subspaces having $\operatorname{dim} U_{1}=\operatorname{dim} Y_{2}$

$$
\begin{array}{ll}
F\left(x^{1}, u^{1}\right) \in T_{x^{1}} X_{1} & G: X_{1} \times U_{1} \rightarrow Y_{1} \\
f\left(x^{2}, u^{2}\right) \in T_{x^{2}} X_{2} & g: X_{2} \times U_{2} \rightarrow Y_{2} .
\end{array}
$$

We always assume $G, g$ are both invertible as in $\S 2 \mathrm{a}$. Equilibrium points $e^{1}$ and $e^{2}$ are defined by

$$
F\left(e^{1}, 0\right)=0 \quad f\left(e^{2}, G\left(e^{1}, 0\right)\right)=0
$$


The composition in Fig. 2.1 acts on state manifolds $X=X_{1} \times X_{2}$, input space $U_{1}$, output space $Y_{2}$ and has state operators:

$$
\begin{aligned}
& \hat{F}\left(\left(x^{1}, x^{2}\right), u\right)=\left(F\left(x^{1}, u\right), f\left(x^{2}, G\left(x^{1}, u\right)\right)\right. \\
& \hat{G}\left(\left(x^{1}, x^{2}\right), u\right)=g\left(x^{2}, G\left(x^{1}, u\right)\right) .
\end{aligned}
$$

These equations are similar to (2.3). Indeed (3.3b) and (2.3b) are exactly the same while (3.3a) and (2.3a) differ only in that $A \hat{F}, F, f$ map one space into a different space.

To characterize (3.3) without reference to $F, G, f, g$ we note that we have a coordinate pair $X=X_{1} \times X_{2}$ of manifold $X_{1}, X_{2}$ for $X$ and the corresponding tangent space $T_{x} X=T_{x^{1}} X_{1}+T_{x^{2}} X_{2}$ decomposition at each $x=\left(x^{1}, x^{2}\right)$. Let $\pi_{x}^{j}$ denote the projection of $T_{x} X$ onto $T_{x} X_{j}$ along $T_{x} X_{k}, k \neq j$. With this notation we get a characterization of factorizations which looks about like the discrete time characterization (2.4) and (2.5). We say that $\left(X_{1}, X_{2}\right)$ is a product coordinate pair for $\hat{F}, \hat{G}$ provided that
(3.4) $\pi_{x}^{1} \hat{F}(x, u)$
is independent of $x^{2}$; here $x=\left(x^{1}, x^{2}\right)$.
$\left(3.4^{\times}\right) \pi_{x}^{2} \hat{F}^{\times}(x, y)$ is independent of $x^{1}$.
(3.5a) $\hat{G}^{I}((a, c), \hat{G}((b, c), u))$ is independent of $c$.
(3.5b) $\hat{G}\left((a, c), \hat{G}^{I}((a, d), y)\right)$ is independent of $a$.

In addition one has

(3.6). If the factors have equilibrium points $e_{1}, e_{2}$, then $e=\left(e_{1}, e_{2}\right)$ satisfies $\hat{F}(e, 0)=0$ and $\hat{F}^{\times}(e, \hat{G}(e, 0))=0$.

Note that not only are these equations similar to those for discrrete time, but Recipe 2.3 for factoring a given system in discrete time makes perfect sense in continuous time. Of course $\pi_{x}^{j}$ replaces $\pi^{j}$. Indeed for continuous time one can prove

THEOREM 3.1. A continuous time system $\hat{F}, \hat{G}$ has a factorization $F, G$ and $f, g$ if it has a product coordinate pair $\left(X_{1}, X_{2}\right)$ with corresponding projections $\pi_{x}^{j}$. Recipe 2.3 again produces the factors. The factors have an equilibrium pair if (3.6) is satisfied.

The converse to Theorem 3.1 is less encumbered in the continuous time case than it is in the discrete time case, since neighborhoods are "preserved by infinitesimal transformations."

THEOREM 3.2. Suppose $\hat{F}, \hat{G}$ with state manifold $X$ factors with systems where state manifolds have dimensions $n$ and $N$ adding to $\hat{N}$, the dimension of $X$. Then 
if $x \in X$ there is a neighborhood $\mathcal{O} \subset X$ of $x$ which has a coordinate pair $\tilde{X}_{1} \times \tilde{X}_{2}$ in the sense that $\tilde{X}_{1} \times \tilde{X}_{2} \supset \mathcal{O}$ and for all $\left(x_{1}, x_{2}\right),(a, c),(b, c)$ and $(a, d)$ in $\mathcal{O}$ equations (3.4), $\left(3.4^{\times}\right)$and (3.5) hold.

The proofs are so close to the discrete time proof in $\S 2$ that we omit it.

We have just seen that minimal factorization of $\hat{F}, \hat{G}$ implied the existence of a product coordinate pair $\left(x_{1}, x_{2}\right)$ for $\hat{F}, \hat{G}$. Now we convert these conditions to infinitesimal form. This converts the problem of finding product coordinate pairs to a problem of solving a certain set of P.D.E.

Suppose we have $\hat{F}, \hat{G}$ and product pair $\left(X_{1}, X_{2}\right)$. The invariance conditions defining product pair can be restated simply in terms of the "invariant distributions" common to nonlinear control. Namely, let $\Delta^{1}$ denote the distribution defined at $x \in X$ by

$$
\Delta_{x}^{2}=T_{x} X_{2}
$$

This indeed associates a subspace of $T_{x} X$ to $x$. Since $\Delta^{2}$ comes from a submanifold of $X$, we know that $\Delta^{2}$ is involutive, that is,

$$
\left[\Delta^{2}, \Delta^{2}\right] \subset \Delta^{2}
$$

c.f. [I, Ch. 1]. However, the invariance (3.3) of $X^{2} \hat{F}$ says precisely that

$$
\left[\hat{F} \Delta^{2}\right] \subset \Delta^{2}
$$

This amounts to a differential equation which $\hat{F}$ and $\Delta^{2}$ must satisfy. Likewise, define $\Delta^{1}$ by

$$
\Delta_{x}^{1}=T_{x} X_{1} .
$$

then

$$
\left[\hat{F}^{x}, \Delta^{1}\right] \subset \Delta^{1} .
$$

Also

$$
\left[\Delta^{1}, \Delta^{1}\right] \subset \Delta^{1} .
$$


We conclude (primarily from $[\mathbf{I}]$ ) that the existence of distributions $\Delta^{1}, \Delta^{2}$ satisfying (3.7), (3.8), (3.10) and (3.11), (3.5) is necessary and sufficient for $\hat{F}, \hat{G}$ to have a product coordinate pair. Thanks are due to Chris Byrnes for for help with this.

\section{§4. UNIQUENESS OF FACTORIZATION}

Since product coordinate pairs correspond to factorization and vice versas, uniqueness of coordinate pairs is the issue here. A leaf of a coordinate pair $X_{1} \times X_{2}$ for $X$ means a set of the form $\left(a, X_{2}\right)$ or $\left(X_{1}, b\right)$; we call these vertical and horizontal leaves, respectively. If $u$ is an input function, that is $u:[0, T] \rightarrow U$ define $\hat{\Phi}(, u)$ to be the map on state space $X$ which implement the dynamics of the differential equation $\frac{d x}{d t}(t)=\hat{F}(x(t), u(t))$. Namely, initialize this differential equation at $x_{0}$; then at time $T$ the solution to the differential equation takes the value $x(T)$; define $\Phi$ by $\hat{\Phi}\left(x_{0}, u\right)=x(T)$. Similarly $\Phi_{1}$ and $\Phi_{2}$ implements the dynamics for $F$ and $f$, respectively. In this article we shall say that a system is reachable if each state $\tilde{x}$ can be reached from any other state $x$ in finite time when the correct input function drives the system. That is $\Phi(x, u)=\tilde{x}$. It is openly reachable if in addition $\Phi(, u)$ is an invertible map real $x$. A key fact is the simple observation.

THEOREM 4.1. Given a continuous time system as in Theorem 3.1 which is openly reachable, any product coordinate pair for the system is uniquely determined by one horizontal and one vertical leaf. This holds for local coordinate pairs as well under a suitably strengthened notion of reachability.

THEOREM 4.2. If a coordinate pair gives rise to a reachable factor $F, G$, then all factors $F, G$ arising from it are reachable. Likewise for $f, g$.

PROOF. Reachability is determined only by $F$ and not influenced by $G$. Formula (2.9) of Recipe 2.3 for $F$ shows that $F$ is completely determined by the coordinate pair and $\hat{F}$. Likewise for $f$ via formula (2.10).

PROOF OF THEOREM 4.1. We start with two leaves $H$ and $V$. Since one is horizontal and one vertical, they intersect in some point $p$. Since $\hat{F}, \hat{G}$ is reachable any point $x \in X$ has the form $\hat{\Phi}(p, u)$ for some input function $u:[0, T] \rightarrow U$. Suppose there is another coordinate pair $\tilde{X}_{1} \times \tilde{X}_{2}$ which contains $H$ and $V$; let $\tilde{L}_{x}$ denote the vertical leaf through $x$. Now $\hat{\Phi}(, u)$ maps each vertical leaf to a subset of a vertical leaf (this is the 
content of Theorem 3.1). Thus $\hat{\Phi}(V, u)$ is contained in $\tilde{L}_{x}$ and also in the vertical leaf $L_{x}$ of $X_{1} \times X_{2}$ through $x$. Leaves $\tilde{L}_{x}$ and $L_{x}$ are manifolds each with dimension equal to the dimension of $V$. If the map $\hat{\Phi}(, u)$ is invertible, near $p$, then $L_{x}=\hat{\Phi}(V, u)=\tilde{L}_{x}$ near $x$.

This argument holds for any $x$, so the vertical leaves $L_{x}$ and $\tilde{L}_{x}$ agree near $x$. An open and closed set argument shows us that all vertical leaves agree.

Horizontal leaves are treated in the same way using $F^{\times}$and $\left(3.4^{\times}\right)$.

Now we apply Theorem 4.1 and use a Poincaré-Dulac approach to show

THEOREM 4.3. Suppose that $\hat{F}, \hat{G}$ is an invertible system as in Theorem 3.1, that it has an equilibrium point $e$, is openly reachable and that the differential equations $\frac{d x}{d t}=\hat{F}(x, 0), \frac{d x}{d t}=\hat{F}^{\times}(x, G(e, 0))$ each have no resonances ${ }^{5}$ at $x=e$. Given two factorizations $F, G, f, g$ and $\tilde{F}, \tilde{G}, \tilde{f}, \tilde{g}$ with equilibrium point $e=\left(e^{1}, e^{2}\right)$ whose state manifolds all have the same dimension. Suppose the linearized systems

$$
\begin{aligned}
D F\left(e^{1}, 0\right) & =D \tilde{F}\left(e^{1}, 0\right) \\
D G\left(e^{1}, 0\right) & =D \tilde{G}\left(e^{1}, 0\right) \\
D f\left(e^{2}, G\left(e^{1}, 0\right)\right) & =D \tilde{f}\left(e^{2}, \tilde{G}\left(e^{1}, 0\right)\right. \\
D g\left(e^{2}, G\left(e^{1}, 0\right)\right) & =D \tilde{g}\left(e^{2}, \tilde{G}\left(e^{1}, 0\right)\right)
\end{aligned}
$$

are equal as shown. Then $\tilde{F}=F$ and $f^{\times}=\tilde{f}^{\times}$in some neighborhood of $e^{1}$ and $e^{2}$.

LEMMA 4.4. (Poincaré-Dulac, see [Har, IX, 12.1]) If $\hat{F}(\cdot, 0)$ has no resonances at $x=e$, then there is a diffeomorphism $\psi, \psi: \mathcal{O} \subset R^{n} \rightarrow X$ on a neighborhood of 0 , with $\psi(0)=e$, so that the map $A: R^{n} \rightarrow R^{n}$ defined at each $s \in \mathcal{O}$ by

$$
A(s)=D_{s} \psi^{-1}(s)[\hat{F}(\psi(s), 0)]
$$

is linear. Note that $A$ and $D_{x} \hat{F}(e, 0)$ are similar.

CONJECTURE 4.5. If $A: R^{n} \rightarrow R^{n}$ has no resonances then each smooth manifold $M$ with $0 \in M$ which is invariant under the flow $e^{\text {At }}$ is actually a linear invariant subspace for $A$.

5 An O.D.E. $\frac{d x}{d t}(t)=\mathcal{A}(x(t))$ with equilibrium points $e$ is said to have no resonances provided evolution operator $D \mathcal{A}(e)$ for the linearized equations has eigenvalues $\lambda_{1}, \ldots, \lambda_{n}$ satisfying equation $m_{1} \lambda_{1}+\cdots+m_{n} \lambda_{n} \neq \lambda_{i}$ for all non-negative integers $m_{k}$. 
A picture is informative.

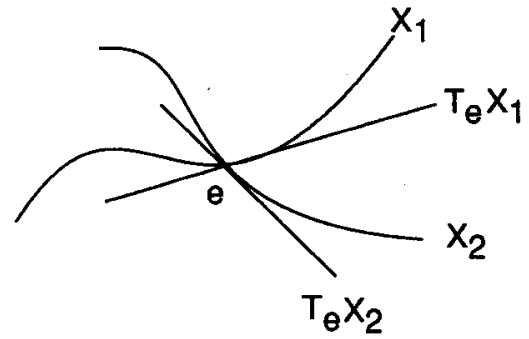

Figure 4.1.

Conjecture 4.5 and Lemma 4.4 says that generically $T_{e} X_{1}$ the tangent space to the $\dot{x}=F^{\times}(x, 0)$ invariant submanifold $X_{1}$ through $e$ determines $X_{1}$ uniquely. Likewise $T_{e} X_{2}$ determined $X_{2}$ uniquely. Theorem 4.1 and Remark 2.3 says that for reachable systems $X_{1}$ and $X_{2}$ determine the dynamical part $F$ and $f^{\times}$of factors uniquely.

PROOF OF THEOREM 4.3. We start with $\hat{F}, \hat{G}$ and two invariant coordinate pairs $X_{1}, X_{2}$ and $\tilde{X}_{1}, \tilde{X}_{2}$. These have horizontal, vertical leaves $H, V$ and $\tilde{H}, \tilde{V}$ through $e$. The hypotheses are set to make the tangent spaces equal:

$$
\begin{aligned}
& T_{e} H=T_{e} \tilde{H} \\
& T_{e} V=T_{e} \tilde{V} .
\end{aligned}
$$

Consider $V$ and $\tilde{V}$. The map $\psi$ of Lemma 4.4 pulls these manifolds back to

$$
V_{0}=\psi^{-1}(V) \quad \tilde{V}_{0}=\psi^{-1}(\tilde{V})
$$

with tangent spaces

$$
T_{0} \tilde{V}_{0}=D \psi^{-1}(e)\left[T_{e} \tilde{V}\right]
$$

etc. This implies

$$
T_{0} V_{0}=T_{0} \tilde{V}_{0}
$$

Also $\psi$ intertwines the flow generated by $\frac{d x}{d t}=\hat{F}(x, 0)$ and the flow generated by $e^{A t}$, so $V_{0}$ and $\tilde{V}_{0}$ are invariant under the $\hat{F}(, 0)$ flow. Lemma 4.5 implies that $V_{0}$ and $\tilde{V}_{0}$ are linear subspaces of $R^{n}$. Consequently $V_{0}=T_{0} V_{0}=T_{0} \tilde{V}_{0}=\tilde{V}_{0}$. Therefore $V=\tilde{V}$.

A similar argument applies to $H$ and $\tilde{H}_{0}$. We have that they are invariant under $F^{\times}(\cdot, G(e, 0))$. There is a map $\mu: R^{n} \rightarrow X$ which composed with these $F^{\times}$"linearized" its action. 
To this point we have shown that the horizontal leaves of $X_{1}, X_{2}$ and of $\tilde{X}_{1}, \tilde{X}_{2}$ through $e$ are equal, so Lemma 4.5 applies directly to give these two coordinate points are actually the same. Finally Remark 2.3 shows that $F$ and $f^{\times}$are determined completely by the coordinate pair. This proves the theorem.

\section{REFERENCES}

[AD] V. Anbantharam and C. A. Desoer, On the stabilization of nonlinear systems, IEEE Trans. Automatic Control, AC-29 (1984), 569-573.

[BGI] H. Bart, I. Gohberg, and M. A. Kaashoek, Minimal Factorization of Matrix and Operator Functions, Birkhäuser, 1979.

[BHI] J. A. Ball and J. W. Helton, Factorization of nonlinear systems: toward a theory for nonlinear $H^{\infty}$ control, Proc. IEEE Conference on Decision and Control, December 1988.

[BH2] J. William Helton, with assistance of Joseph A. Ball, Charles R. Johnson, and John Palmer, Operator theory, analytic functions, matrices, and electrical engineering, Expository Lectures from the CBMS Regional Conference held at Lincoln, Nebraska, August 1985 (Regional conference series in mathematics, ISSN 0160-7642; no. 68, 1987).

[Har] P. Hartman, Ordinary Differential Equations, Birkhäuser, Boston, 1982.

[Hm] J. Hammer, Nonlinear systems: stabilization and coprimeness, International J. Control, 42, no. 1 (1985), pp. 1-20.

[I] A. Isadori, Nonlinear control systems: an introduction, Springer Lecture Notes in Control and Information Science, no. 72, 1985.

Department of Mathematics (C-012)

University of California, San Diego

La Jolla, California 92093 U.S.A. 